\title{
Null-Controllable Set Computation for a Class of Constrained Bilinear Systems
}

\author{
Moritz Schulze Darup and Martin Mönnigmann
}

\begin{abstract}
We present a method for the exact computation of null-controllable sets for single-input bilinear systems with input and state constraints. The proposed approach is based on first linearizing the bilinear system exactly, and applying known methods for the recursive calculation of null-controllable sets subsequently. While these steps are obvious from a conceptual point of view, it has to be taken into account that the constraints are transformed in the exact linearization step. Unfortunately, the transformed constraints are in general non-convex, even if the original constraints are convex. We show how to represent the transformed constraints in terms of a finite number of convex sets, which is instrumental for the computer-based evaluation of the null-controllable sets.
\end{abstract}

\section{INTRODUCTION}

The analysis of controllability has a long history in control theory. For linear unconstrained systems, Hautus [6] derived the well-known algebraic criterion for the detection of global null-controllability in 1970. Gutman and Cwikel [5] as well as Keerthi and Gilbert [8] provided algorithms for the iterative but exact computation of null-controllable sets for linear systems with input and state constraints. The computation of null-controllable sets for nonlinear constrained systems is considerably more difficult than for linear constrained systems. Consequently, approaches for nonlinear systems usually involve approximations (see, e.g., [1]).

We present an algorithm for the exact computation of nullcontrollable sets for a particular, admittedly special, class of nonlinear constrained systems. Specifically, we consider single-input bilinear systems [2], [4] of the form

$$
x(k+1)=A x(k)+(b+N x(k)) u(k), \quad x(0)=x_{0},
$$

with input and state constraints

$$
u(k) \in \mathcal{U} \subset \mathbb{R}, \quad x(k) \in \mathcal{X} \subset \mathbb{R}^{n}, \quad \forall k \in \mathbb{N},
$$

where $A, N \in \mathbb{R}^{n \times n}$ and $b \in \mathbb{R}^{n}$. The sets $\mathcal{U}$ and $\mathcal{X}$ are restricted to be convex polytopes with the origin in their interiors. A sequence of inputs is called admissible, if all its elements and the resulting trajectory of (1) respect (2). We calculate the $i$-step null-controllable set $\mathcal{N}_{i}$, i.e., the set of all states $x_{0} \in \mathcal{X}$ that can be steered to the origin with an admissible input sequence that is at most $i \in \mathbb{N}$ steps long. It is the main idea behind the proposed algorithm to reformulate system (1), (2) in terms of a linearized system resulting from exact linearization [7]. We then use wellknow algorithms for linear constrained systems [5], [8] to calculate null-controllable sets for (1), (2). Unfortunately,

Both authors are with the Dep. of Mechanical Engineering, RuhrUniversität Bochum. E-mail: moritz.schulzedarup@rub.de and martin.moennigmann@rub.de. the state and input transformation required for the exact linearization result in a more complicated description of the constraints (2). However, we show that the transformed constraints can be represented by a finite set of convex constraints, which is instrumental for the exact computation of the null-controllable sets.

We state basic notation and preliminaries in Sect. II. Section III deals with the exact linearization of bilinear system. The main results of the paper, i.e. the reformulation of the transformed constraints and the design of an algorithm for the exact computation of null-controllable sets, are treated in Sect. IV. Finally, Sects. V and VI present two illustrative examples and state conclusions, respectively.

\section{Notation AND PRELIMINARIES}

We denote matrices by capital letters, vectors and scalars with lowercase letters and sets with calligraphic letters. Let $A \in \mathbb{R}^{n \times n}$ and $B \in \mathbb{R}^{n \times m}$. By $B^{T}, \operatorname{rk}(B)$ and $\operatorname{cs}(B)$ denote the transpose, rank and column space of the matrix $B$. For $\nu \in \mathbb{N}$, define $C_{\nu}(A, B) \in \mathbb{R}^{n \times \nu(n \cdot m)}$ recursively by $C_{\nu}(A, B):=\left[C_{\nu-1}(A, B) \quad A^{\nu-1} B\right]$, where $C_{0}(A, B):=[]$ is defined to be an empty matrix. Let $I_{n} \in \mathbb{R}^{n \times n}$ and $e_{i} \in \mathbb{R}^{n}$ refer to the identity matrix and the $i$-th canonical basis vector, respectively. Let $\mathcal{A} \subseteq \mathbb{R}^{n}$ and $\mathcal{C} \subseteq \mathbb{R}^{m}$. By $\mathcal{A}^{\perp}$ denote the orthogonal complement of $\mathcal{A}$. Furthermore, we define the sets $B \mathcal{C}:=\{B c \mid c \in \mathcal{C}\}$ and $B^{-1} \mathcal{A}:=\left\{c \in \mathbb{R}^{m} \mid B c \in \mathcal{A}\right\}$. Recall that $B^{-1} \mathcal{A}$ equals $\hat{B} \mathcal{A}$ with $\hat{B}=B^{-1}$, if $B$ is invertible, but $B^{-1} \mathcal{A}$ is well defined even if $B$ is not invertible. $L_{f}^{i} h(x)$ denotes the $i$-th Lie derivative of $h$ along $f$, where $L_{f}^{0} h(x)=h(x)$ (see [7] for details). Finally, define $\mathbb{N}_{i}^{k}:=\{j \in \mathbb{N} \mid i \leq j \leq k\}$.

\section{A. Null-Controllable Set Computation for Linear Systems}

For $N=0$ the bilinear system (1) degenerates into a linear system of the form $x(k+1)=A x(k)+b u(k)$. Gutman and Cwikel [5] introduced an iterative procedure for calculating $i$-step null-controllable sets for linear systems subject to constraints (2). Starting with $\mathcal{N}_{0}=\{0\}$, the recursion

$$
\mathcal{N}_{i+1}=\left\{x \in \mathcal{X} \mid \exists u \in \mathcal{U}: A x+b u \in \mathcal{N}_{i}\right\}
$$

results in a nested sequence $\left(\mathcal{N}_{i} \subseteq \mathcal{N}_{i+1}\right)$ of null-controllable sets [5]. Keerthi and Gilbert [8] provided a computationally efficient algorithm to evaluate $\mathcal{N}_{i+1}$ based on projections. In this context, let the extended state and the associated constraints be defined by

$$
z:=\left[\begin{array}{l}
x \\
u
\end{array}\right] \text { and } \mathcal{Z}:=\{z \mid x \in \mathcal{X}, u \in \mathcal{U}\},
$$


respectively. Then, the recursion (3) can be carried out according to [8]

$$
\begin{aligned}
\mathcal{N}_{i+1} & =P\left\{z \in \mathcal{Z} \mid S z \in \mathcal{N}_{i}\right\} \\
& =P\left(S^{-1} \mathcal{N}_{i} \cap \mathcal{Z}\right),
\end{aligned}
$$

where $P, S \in \mathbb{R}^{n \times(n+1)}$ with $P:=\left[\begin{array}{ll}I_{n} & 0\end{array}\right]$ and $S:=$ $\left[\begin{array}{ll}A & b\end{array}\right]$. We state the following remark for later use. Note that $\mathcal{Z}$ is convex, since $\mathcal{X}$ and $\mathcal{U}$ are convex sets by assumption and recall that a projection is a particular linear transformation.

Remark 1: The sets $\mathcal{N}_{i+1}$ are convex for linear systems. This follows from (5), since intersections of convex sets are convex sets, and since $B \mathcal{A}$ and $B^{-1} \mathcal{A}$ are convex sets for every linear transformation $B$ and every convex set $\mathcal{A}$ (see, e.g., [10], Thms. 2.1 and 3.4).

\section{B. Exact Linearization of Input Affine Systems}

Consider an input affine system of the form

$$
x(k+1)=f(x(k))+g(x(k)) u(k), \quad y(k)=h(x(k)) .
$$

System (6) is said to have relative degree $r$ at $x^{*}$, if the following conditions hold [7]:

$$
\begin{aligned}
L_{g} L_{f}^{i} h(x) & =0 \quad \text { for all } x \text { in a neighborhood of } x^{*} \\
L_{g} L_{f}^{r-1} h\left(x^{*}\right) & \neq 0 .
\end{aligned}
$$

The relative degree can be used to determine whether there exists an exact state space linearization to (6). This is stated more concisely in Lemma 1 below. If an exact linearization exists, the transformed system has the form

$$
\tilde{x}(k+1)=\tilde{A} \tilde{x}(k)+\tilde{b} \tilde{u}(k),
$$

where

$$
\tilde{A}=\left[\begin{array}{cc}
0 & I_{n-1} \\
0 & 0
\end{array}\right] \text { and } \quad \tilde{b}=e_{n} .
$$

Lemma 1 (cf. Lem. 4.2.1 in [7]): The state space system (6) can be transformed into the linear system (9) in a neighborhood of $x^{*}$, if and only if (6) has relative degree $r=n$ at $x^{*}$.

If the condition $r=n$ is satisfied at $x^{*}$, the transformation from (6) to (9) is given by $u=\alpha(x)(\tilde{u}-\beta(x))$ and $\tilde{x}=\gamma(x)$, where

$$
\begin{aligned}
\alpha(x) & =\frac{1}{L_{g} L_{f}^{n-1} h(x)}, \quad \beta(x)=L_{f}^{n} h(x), \\
\gamma_{i}(x) & =L_{f}^{i-1} h(x), \quad i=1, \ldots, n .
\end{aligned}
$$

This transformation is in general nonlinear.

\section{EXACT LINEARIZATION OF BILINEAR SYSTEMS}

The bilinear system (1) obviously belongs the system class (6) for $f(x)=A x$ and $g(x)=b+N x$. We restrict ourselves to linear outputs of the form

$$
y(k)=h(x(k))=c^{T} x(k),
$$

where $c \in \mathbb{R}^{n}$. This restriction is imposed in order to keep (11) and (12) tractable. We collect some conditions in the following assumption for ease of reference.
Assumption 1: There exists a linear output (13) for the bilinear system (1) such that the resulting SISO system has relative degree $r=n$ for all $x^{*} \in \mathcal{X}^{*}$ with

$$
\mathcal{X}^{*}=\left\{x \in \mathbb{R}^{n} \mid b+N x \neq 0\right\} .
$$

The Lie derivatives and conditions (7), (8) read

$$
L_{f}^{i} h(x)=c^{T} A^{i} x \quad \text { and } \quad L_{g} L_{f}^{i} h(x)=c^{T} A^{i}(b+N x),
$$

and

$$
\begin{aligned}
c^{T} A^{i}(b+N x)=0 & \forall x \in \mathbb{R}^{n} \text { and } \forall i \in \mathbb{N}_{0}^{n-2}, \\
c^{T} A^{n-1}\left(b+N x^{*}\right) \neq 0 & \forall x^{*} \in \mathcal{X}^{*},
\end{aligned}
$$

respectively, for a bilinear system (1) that satisfies Assumption 1. Note that the relative degree is not defined for all $x^{*}$ that are not in $\mathcal{X}^{*}$. For every $x^{*} \in \mathcal{X}^{*}$, the exact linearization (9) results for the linearizing input transformation

$$
u=\frac{1}{c^{T} A^{n-1}(b+N x)}\left(\tilde{u}-c^{T} A^{n} x\right)
$$

and the linear coordinate transformation $\tilde{x}=\gamma(x)=M x$, where

$$
M=C_{n}^{T}\left(A^{T}, c\right)
$$

equals the observability matrix for linear systems. Since the transformation $x=M^{-1} \tilde{x}$ is linear, it turns out to be convenient to express (9) in the original state $x$ instead of $\tilde{x}$. The resulting system reads

$$
x(k+1)=\hat{A} x(k)+\hat{b} \hat{u}(k),
$$

where

$$
\hat{A}=M^{-1} \tilde{A} M, \quad \hat{b}=M^{-1} \tilde{b} \quad \text { and } \quad \hat{u}=\tilde{u} .
$$

Systems (9) and (19) are one to one, since $M$ is invertible.

The exact linearization obviously hinges on Assumption 1. Proposition 1 given below states a necessary condition for Assumption 1 to hold. Unfortunately, the class of tractable bilinear systems is small as illustrated in Lem. 2 further below.

Proposition 1: Let $B:=\left[\begin{array}{ll}b & N\end{array}\right]$ and let $\mathcal{X}^{*}$ be as in (14). Assume there exists a $c \in \mathbb{R}$ for the binlinear system (1) such that the SISO system with output $y=c^{T} x(k)$ has relative degree $r=n$ for all $x^{*} \in \mathcal{X}^{*}$. Then

$$
\operatorname{rk}\left(C_{n-1}(A, B)\right)<\operatorname{rk}\left(C_{n}(A, B)\right) .
$$

Proof: There exists a $c$ such that the SISO system with output $y=c^{T} x(k)$ has relative degree $r=n$ for all $x^{*} \in \mathcal{X}^{*}$ if and only if (15), (16) hold. Since the 1.h.s. in (15) can be written as

$$
c^{T} A^{i}(b+N x)=c^{T} A^{i} B\left[\begin{array}{l}
1 \\
x
\end{array}\right],
$$

condition (15) holds for all $x \in \mathbb{R}^{n}$ if and only if

$$
c A^{i} B=0 \text { for all } i \in \mathbb{N}_{0}^{n-2}
$$

It remains to apply (22) to (16). This yields

$$
c^{T} A^{n-1} B\left[\begin{array}{c}
1 \\
x^{*}
\end{array}\right] \neq 0 \Rightarrow c^{T} A^{n-1} B \neq 0,
$$


where the converse does in general not hold. Note that Prop. 1 essentially states only a necessary condition, because (24) is only an implication but not an equivalence. Relation (23) and the relation on the r.h.s. of (24) can equivalently be written as

$$
\begin{gathered}
c^{T} C_{n-1}(A, B)=0 \quad \text { and } \quad c^{T} A^{n-1} B \neq 0, \text { or } \\
c \in \operatorname{cs}\left(C_{n-1}(A, B)\right)^{\perp} \quad \text { and } \quad c \notin \operatorname{cs}\left(A^{n-1} B\right)^{\perp} .
\end{gathered}
$$

We complete the proof by showing that (26) implies

$$
\operatorname{cs}\left(C_{n-1}(A, B)\right) \subset \operatorname{cs}\left(C_{n}(A, B)\right)
$$

which in turn implies the claim (21). To show (26) $\Longrightarrow$ (27) assume (26) holds, assume (27) does not hold, and show a contradiction results. If (27) does not hold, we have the first of the following two relations

$$
\begin{aligned}
\operatorname{cs}\left(C_{n-1}(A, B)\right) & \supseteq \operatorname{cs}\left(C_{n}(A, B)\right) \\
& =\operatorname{cs}\left(\left[C_{n-1}(A, B) A^{n-1} B\right]\right)
\end{aligned}
$$

and the second relation holds by definition of $C_{\nu}(A, B)$ (cf. Sect. II). Relation (29) implies $\operatorname{cs}\left(A^{n-1} B\right) \subseteq$ $\operatorname{cs}\left(C_{n-1}(A, B)\right)$, from which we infer $\operatorname{cs}\left(A^{n-1} B\right)^{\perp} \supseteq$ $\operatorname{cs}\left(C_{n-1}(A, B)\right)^{\perp}$, which in turn yields

$$
c \in \operatorname{cs}\left(C_{n-1}(A, B)\right)^{\perp} \Rightarrow c \in \operatorname{cs}\left(A^{n-1} B\right)^{\perp}
$$

The last statement contradicts (26), which completes the proof.

Lemma 2: A necessary condition for (21) to be fulfilled is $\operatorname{rk}(B)=1$.

Proof: It is well-known from linear systems theory (see e.g. [3], p. 151) that $\operatorname{rk}\left(S_{\nu}(A, B)\right)=\operatorname{rk}\left(S_{n}(A, B)\right)$ for all

$$
\nu \geq n+1-\operatorname{rk}(B) \text {. }
$$

In order to satisfy (21), we must have $n+1-\operatorname{rk}(B)>n-1$ or equivalently $\operatorname{rk}(B)<2$. On the other hand (21) implies $\operatorname{rk}(B)>0$.

\section{Null-Controllable Set Computation for BILINEAR SYSTEMS}

In principle, the computation of null-controllable sets for bilinear systems can be carried out analogously to the procedure in the linear case. The recursion (3) for linear systems has to be replaced by

$$
\mathcal{N}_{i+1}=\left\{x \in \mathcal{X} \mid \exists u \in \mathcal{U}: A x+(b+N x) u \in \mathcal{N}_{i}\right\},
$$

with $\mathcal{N}_{0}=\{0\}$ in the bilinear case. It is possible to adapt the projection method (4), (5) to the bilinear case by using the exact linearization (19). This will be shown in the remainder of the section. As a preparation we show that (19) is not only useful for all $x \in \mathcal{X}^{*}$ but all $x \in \mathbb{R}^{n}$ in the following lemma.

Lemma 3: Let $c \in \mathbb{R}^{n}$ be such that conditions (15), (16) hold for the bilinear system (1), (2) and let $\hat{A}, \hat{b}$ be as in (19). Define $\varphi: \mathbb{R}^{n} \times \mathbb{R} \rightarrow \mathbb{R}$ by

$$
\varphi(x, u):=c^{T} A^{n-1}(b+N x) u+c^{T} A^{n} x .
$$

Then

$$
A x+(b+N x) u=\hat{A} x+\hat{b} \varphi(x, u),
$$

for all $x \in \mathbb{R}^{n}$ and all $u \in \mathbb{R}$.

Proof: Let $x \in \mathcal{X}^{*}$ at first, where $\mathcal{X}^{*}$ is defined as in (14). Rearranging (17) yields $\tilde{u}=\varphi(x, u)$, where we use that (17) is well defined, since $x \in \mathcal{X}^{*}$ implies $c^{T} A^{n-1}(b+$ $N x) \neq 0$. We infer from (19) and the last equation in (20) that $x(k+1)=\hat{A} x(k)+\hat{b} \varphi(x(k), u(k))$. On the other hand $x(k+1)=A x(k)+(b+N x(k)) u(k)$ according to (1), therefore the claim holds for all $x \in \mathcal{X}^{*}$ and all $u \in \mathbb{R}$.

Now let $x \in \mathbb{R}^{n} \backslash \mathcal{X}^{*}$. According to the definition of $\mathcal{X}^{*}$ in (14), $x \in \mathbb{R}^{n} \backslash \mathcal{X}^{*}$ is equivalent to $b+N x=0$, therefore

$$
\varphi(x, u)=c^{T} A^{n} x .
$$

Substituting $b+N x=0$ and (33) into (32) yields

$$
A=\hat{A}+\hat{b} c^{T} A^{n} \text {. }
$$

Thus, (32) holds for all $x \in \mathbb{R}^{n} \backslash \mathcal{X}^{*}$ if and only if (34) holds. Equation (34) can be proved by substituting the coordinate transformation (20), the definition of $\tilde{A}$ and $\tilde{b}$ from (10) and the definition of $M$ from (18) into the r.h.s. of (34).

It remains to state the original constraints (2) in terms of the variables $x$ and $\hat{u}=\varphi(x, u)$ of the linearized system (19). Let

$\hat{z}:=\left[\begin{array}{l}x \\ \hat{u}\end{array}\right]$ and $\hat{\mathcal{Z}}:=\{\hat{z} \mid \hat{u}=\varphi(x, u), x \in \mathcal{X}, u \in \mathcal{U}\}$

Then, by definition, we have $x \in \mathcal{X}, u \in \mathcal{U}$ if and only if $\hat{z} \in \hat{\mathcal{Z}}$. The following lemma provides a more detailed description of $\hat{\mathcal{Z}}$.

Lemma 4: The set $\hat{\mathcal{Z}}$ defined in (35) can be expressed as $\hat{\mathcal{Z}}=\hat{\mathcal{Z}}^{\prime} \cup \hat{\mathcal{Z}}^{\prime \prime}$, where

$$
\begin{aligned}
\hat{\mathcal{Z}}^{\prime} & =\left\{\hat{z} \mid x \in \mathcal{X} \cap \mathcal{X}^{*}, \frac{\hat{u}-c^{T} A^{n} x}{c^{T} A^{n-1}(b+N x)} \in \mathcal{U}\right\}, \\
\hat{\mathcal{Z}}^{\prime \prime} & =\left\{\hat{z} \mid x \in \mathcal{X} \backslash \mathcal{X}^{*}, \hat{u}=c^{T} A^{n} x\right\} .
\end{aligned}
$$

Proof: Since $\mathcal{X}=\left(\mathcal{X} \cap \mathcal{X}^{*}\right) \cup\left(\mathcal{X} \backslash \mathcal{X}^{*}\right)$, we may rewrite (35) as the union

$$
\begin{aligned}
\hat{\mathcal{Z}}= & \left\{\hat{z} \mid \hat{u}=\varphi(x, u), x \in \mathcal{X} \cap \mathcal{X}^{*}, u \in \mathcal{U}\right\} \cup \\
& \left\{\hat{z} \mid \hat{u}=\varphi(x, u), x \in \mathcal{X} \backslash \mathcal{X}^{*}, u \in \mathcal{U}\right\} .
\end{aligned}
$$

Consider the case $x \in \mathcal{X} \cap \mathcal{X}^{*}$ first. In this case, the input transformation (17) applies and, with $\hat{u}=\tilde{u}$ from (20), we have $u=\left(\hat{u}-c^{T} A^{n} x\right) /\left(c^{T} A^{n-1}(b+N x)\right)$. Upon substituting this expression for $u$ into the r.h.s. of (36) $\hat{\mathcal{Z}}^{\prime}$ results. If, on the other hand, $x \in \mathcal{X} \backslash \mathcal{X}^{*}$, then equation (33) implies that the second set on the r.h.s. of (36) is equal to $\hat{\mathcal{Z}}^{\prime \prime}$.

At first sight, it is surprising that the set $\mathcal{U}$ from the input constraints does not appear in the definition of the set $\hat{\mathcal{Z}}^{\prime \prime}$. This is due to the fact that $c^{T} A^{n-1}(b+N x)=0$ for all $x \notin \mathcal{X}^{*}$. Hence, according to (31), the original input $u$ has no effect on the artificial input $\hat{u}$ and we may choose $u=0$ to satisfy the constraints $\mathcal{U}$.

Having specified $\hat{\mathcal{Z}}$, null-controllable sets for the bilinear system (1), (2) can be computed with the recursion

$$
\mathcal{N}_{i+1}=P\left(\left[\begin{array}{ll}
\hat{A} & \hat{b}
\end{array}\right]^{-1} \mathcal{N}_{i} \cap \hat{\mathcal{Z}}\right),
$$

which is the analogue of (5) for bilinear systems. Unfortunately, it is not obvious how to apply (37) in a computational 
procedure, since $\hat{\mathcal{Z}}$ is in general not convex. We try to reformulate $\hat{\mathcal{Z}}$ (and later $\mathcal{N}_{i+1}$ ) in terms of the union of convex sets as a remedy. First note that the polytopic constraints (2) can be expressed as

$$
\mathcal{U}=\left\{u \in \mathbb{R} \mid h_{u} u \leq d_{u}\right\}, \mathcal{X}=\left\{x \in \mathbb{R}^{n} \mid H_{x} x \leq d_{x}\right\},
$$

where $h_{u}, d_{u} \in \mathbb{R}^{2}, H_{x} \in \mathbb{R}^{p \times n}, d_{x} \in \mathbb{R}^{p}$, and $p \in \mathbb{N}$ refers to the number of hyperplanes required to state the constraints $\mathcal{X}$. Note that $\mathcal{U}$ can also be stated as $\mathcal{U}=\{u \in \mathbb{R} \mid \underline{u} \leq u \leq$ $\bar{u}\}$ in the single-input case, where $\underline{u}, \bar{u} \in \mathbb{R}$ and $\underline{u}<0<\bar{u}$. A comparison of the interval $\mathcal{U}$ and (38) yields

$$
h_{u}=\left[\begin{array}{r}
1 \\
-1
\end{array}\right] \quad \text { and } \quad d_{u}=\left[\begin{array}{r}
\bar{u} \\
-\underline{u}
\end{array}\right] \text {, }
$$

which we note for later use. The set $\hat{\mathcal{Z}}$ can now be expressed as a union of convex polytopes as detailed in Lemma 5.

Lemma 5: Let $\mathcal{U}$ and $\mathcal{X}$ be convex polytopes of the form (38). Then, the set $\hat{\mathcal{Z}}$ as defined in (35) can be expressed as the union $\hat{\mathcal{Z}}=\hat{\mathcal{Z}}^{1} \cup \hat{\mathcal{Z}}^{2}$ of the two convex polytopes

$$
\begin{aligned}
& \hat{\mathcal{Z}}^{1}=\left\{\hat{z} \in \mathbb{R}^{n+1} \mid H_{\hat{z}}^{(+)} \hat{z} \leq d_{\hat{z}}^{(+)}\right\} \quad \text { and } \\
& \hat{\mathcal{Z}}^{2}=\left\{\hat{z} \in \mathbb{R}^{n+1} \mid H_{\hat{z}}^{(-)} \hat{z} \leq d_{\hat{z}}^{(-)}\right\},
\end{aligned}
$$

where

$$
\begin{aligned}
H_{\hat{z}}^{( \pm)} & =\left[\begin{array}{cc}
H_{x} & 0 \\
\mp c^{T} A^{n-1} N & 0 \\
\mp h_{u} c^{T} A^{n} \mp d_{u} c^{T} A^{n-1} N & \pm h_{u}
\end{array}\right], \\
d_{\hat{z}}^{( \pm)} & =\left[\begin{array}{c}
d_{x} \\
\pm c^{T} A^{n-1} b \\
\pm d_{u} c^{T} A^{n-1} b
\end{array}\right] .
\end{aligned}
$$

Proof: We prove the claim by showing that $\hat{\mathcal{Z}}^{1} \cup \hat{\mathcal{Z}}^{2}=$ $\hat{\mathcal{Z}}^{\prime} \cup \hat{\mathcal{Z}}^{\prime \prime}$, where $\hat{\mathcal{Z}}^{\prime}$ and $\hat{\mathcal{Z}}^{\prime \prime}$ are as in Lem. 4 . First note that $\hat{z}=\left(\begin{array}{ll}x^{T} & \hat{u}\end{array}\right)^{T} \in \hat{\mathcal{Z}}^{\prime}$ if and only if

$$
\begin{aligned}
H_{x} x & \leq d_{x}, \\
b+N x & \neq 0, \\
h_{u}\left(\frac{\hat{u}-c^{T} A^{n} x}{c^{T} A^{n-1}(b+N x)}\right) & \leq d_{u} .
\end{aligned}
$$

According to (16), we have

$$
b+N x \neq 0 \quad \Leftrightarrow \quad c^{T} A^{n-1}(b+N x) \neq 0 .
$$

Since $c^{T} A^{n-1}(b+N x)$ is a real number, condition (45) is equivalent to

$$
c^{T} A^{n-1}(b+N x)>0 \text { or } c^{T} A^{n-1}(b+N x)<0 .
$$

$\hat{\mathcal{Z}}^{\prime}$ can be written as the union $\hat{\mathcal{Z}}^{\prime}=\hat{\mathcal{Z}}^{\prime \prime \prime} \cup \hat{\mathcal{Z}}^{\prime \prime \prime \prime}$, where $\hat{\mathcal{Z}}^{\prime \prime \prime}$ and $\hat{\mathcal{Z}}^{\prime \prime \prime \prime}$ are described by the inequalities

$$
\begin{aligned}
H_{x} x & \leq d_{x}, \\
c^{T} A^{n-1}(b+N x) & >0, \\
h_{u}\left(\hat{u}-c^{T} A^{n} x\right) & \leq d_{u} c^{T} A^{n-1}(b+N x)
\end{aligned}
$$

if the 1.h.s. in (48) applies, and

$$
\begin{aligned}
H_{x} x & \leq d_{x}, \\
c^{T} A^{n-1}(b+N x) & <0, \\
h_{u}\left(\hat{u}-c^{T} A^{n} x\right) & \geq d_{u} c^{T} A^{n-1}(b+N x),
\end{aligned}
$$

if the r.h.s. in (48) applies. Note that the last inequality in (49) (resp. (50)) results from the multiplication with the positive (resp. negative) value $c^{T} A^{n-1}(b+N x)$. A careful inspection of the coefficients in (49) and (50) shows that we found the matrices $H_{\hat{z}}^{( \pm)}$and $d_{\hat{z}}^{( \pm)}$, where "+" and "-" refer to (49) and (50), respectively. However, $\hat{\mathcal{Z}}^{\prime \prime \prime} \subset \hat{\mathcal{Z}}^{1}$ and $\hat{\mathcal{Z}}^{\prime \prime \prime \prime} \subset \hat{\mathcal{Z}}^{2}$, because there appear strict inequalities in (49) and (50), where non-strict inequalities apply in the definition of $\hat{\mathcal{Z}}^{1}$ and $\hat{\mathcal{Z}}^{2}$. More precisely, $\hat{\mathcal{Z}}^{1}$ is defined by the non-strict inequalities

$$
\begin{aligned}
H_{x} x & \leq d_{x}, \\
c^{T} A^{n-1}(b+N x) & \geq 0, \\
h_{u}\left(\hat{u}-c A^{n} x\right) & \leq d_{u} c^{T} A^{n-1}(b+N x)
\end{aligned}
$$

A comparison of (49) and (51) shows that the elements of $\hat{\mathcal{Z}}^{1} \backslash \hat{\mathcal{Z}}^{\prime \prime \prime}$ obey

$$
\begin{aligned}
H_{x} x & \leq d_{x}, \\
c^{T} A^{n-1}(b+N x) & =0 \\
h_{u}\left(\hat{u}-c^{T} A^{n} x\right) & \leq 0,
\end{aligned}
$$

where 0 on the 1.h.s. of the last inequality results from $c^{T} A^{n-1}(b+N x)=0$. Moreover, due to the special structure of $h_{u}$ as specified in (39), we have

$$
h_{u}\left(\hat{u}-c^{T} A^{n} x\right) \leq 0 \quad \Leftrightarrow \quad \hat{u}-c^{T} A^{n} x=0 .
$$

Finally, from (47) we infer that

$$
c^{T} A^{n-1}(b+N x)=0 \quad \Leftrightarrow \quad b+N x=0 .
$$

Using (53) and (54), we may rewrite (52) as

$$
\begin{aligned}
H_{x} x & \leq d_{x}, \\
b+N x & =0, \\
\hat{u}-c^{T} A^{n} x & =0,
\end{aligned}
$$

which is the set of conditions that defines $\hat{\mathcal{Z}}^{\prime \prime}$ as introduced in Lemma 4. This implies $\hat{\mathcal{Z}}^{\prime \prime}=\hat{\mathcal{Z}}^{1} \backslash \hat{\mathcal{Z}}^{\prime \prime \prime}$, therefore

$$
\hat{\mathcal{Z}}^{1}=\left(\hat{\mathcal{Z}}^{1} \backslash \hat{\mathcal{Z}}^{\prime \prime \prime}\right) \cup \hat{\mathcal{Z}}^{\prime \prime \prime}=\hat{\mathcal{Z}}^{\prime \prime} \cup \hat{\mathcal{Z}}^{\prime \prime \prime} \text {. }
$$

Analogously, it can be shown that $\hat{\mathcal{Z}}^{2}=\hat{\mathcal{Z}}^{\prime \prime} \cup \hat{\mathcal{Z}}^{\prime \prime \prime \prime}$ holds. These statements complete the proof, since we have

$$
\begin{aligned}
\hat{\mathcal{Z}} & =\hat{\mathcal{Z}}^{\prime} \cup \hat{\mathcal{Z}}^{\prime \prime}=\hat{\mathcal{Z}}^{\prime \prime} \cup \hat{\mathcal{Z}}^{\prime \prime \prime} \cup \hat{\mathcal{Z}}^{\prime \prime \prime \prime} \\
& =\left(\hat{\mathcal{Z}}^{\prime \prime} \cup \hat{\mathcal{Z}}^{\prime \prime \prime}\right) \cup\left(\hat{\mathcal{Z}}^{\prime \prime} \cup \hat{\mathcal{Z}}^{\prime \prime \prime \prime}\right)=\hat{\mathcal{Z}}^{1} \cup \hat{\mathcal{Z}}^{2}
\end{aligned}
$$

We pointed out above that the sets $\mathcal{N}_{i+1}$ are in general not convex for bilinear systems. Just as for $\hat{\mathcal{Z}}$, a representation of the sets $\mathcal{N}_{i+1}$ in terms of convex sets can be found. This is summarized in the following lemma.

Lemma 6: Let $\hat{\mathcal{Z}}^{1}$ and $\hat{\mathcal{Z}}^{2}$ be defined as in Eqs. (40)(43). Assume there exist convex sets $\mathcal{N}_{i}^{1}, \ldots, \mathcal{N}_{i}^{l}$ such that $\mathcal{N}_{i}=\bigcup_{j=1}^{l} \mathcal{N}_{i}^{j}$. Define the sets

$$
\begin{aligned}
& \mathcal{N}_{i+1}^{2 j-1}=P\left(\left[\begin{array}{ll}
\hat{A} & \hat{b}
\end{array}\right]^{-1} \mathcal{N}_{i}^{j} \cap \hat{\mathcal{Z}}^{1}\right) \text { and } \\
& \mathcal{N}_{i+1}^{2 j}=P\left(\left[\begin{array}{ll}
\hat{A} & \hat{b}
\end{array}\right]^{-1} \mathcal{N}_{i}^{j} \cap \hat{\mathcal{Z}}^{2}\right)
\end{aligned}
$$

for $j=1, \ldots, l$. Then, for every $j \in\{1, \ldots, 2 l\}, \mathcal{N}_{i+1}^{j}$ is a convex set and

$$
\mathcal{N}_{i+1}=\bigcup_{j=1}^{l} \mathcal{N}_{i+1}^{2 j-1} \cup \mathcal{N}_{i+1}^{2 j} .
$$


for $\mathcal{N}_{i+1}$ as specified in (37).

Proof: The sets $\mathcal{N}_{i+1}^{2 j-1}$ and $\mathcal{N}_{i+1}^{2 j}$ can be shown to be convex using the arguments from Rem. 1 , since $\mathcal{N}_{i}^{1}, \ldots, \mathcal{N}_{i}^{l}$ and $\hat{\mathcal{Z}}^{1}, \hat{\mathcal{Z}}^{2}$ are convex by assumption and due to Lem. 5 , respectively. In order to prove relation (58), we have to show that the r.h.s. in (37) and (58) are equal. This can be seen from from the following chain of equalities, which are commented below. Let $\tilde{S}=\left[\begin{array}{ll}\hat{A} & \hat{b}\end{array}\right]^{-1}$ for short.

$$
\begin{aligned}
P\left(\tilde{S} \mathcal{N}_{i} \cap \hat{\mathcal{Z}}\right) & =P\left(\tilde{S}\left(\mathcal{N}_{i}^{1} \cup \cdots \cup \mathcal{N}_{i}^{l}\right) \cap\left(\hat{\mathcal{Z}}^{1} \cup \hat{\mathcal{Z}}^{2}\right)\right) \\
& =P\left(\left(\tilde{S} \mathcal{N}_{i}^{1} \cup \cdots \cup \tilde{S} \mathcal{N}_{i}^{l}\right) \cap\left(\hat{\mathcal{Z}}^{1} \cup \hat{\mathcal{Z}}^{2}\right)\right) \\
& =P\left(\left(\tilde{S} \mathcal{N}_{k}^{1} \cap \hat{\mathcal{Z}}^{1}\right) \cup \cdots \cup\left(\tilde{S} \mathcal{N}_{i}^{l} \cap \hat{\mathcal{Z}}^{2}\right)\right) \\
& =P\left(\tilde{S} \mathcal{N}_{k}^{1} \cap \hat{\mathcal{Z}}^{1}\right) \cup \cdots \cup P\left(\tilde{S} \mathcal{N}_{i}^{l} \cap \hat{\mathcal{Z}}^{2}\right) \\
& =\mathcal{N}_{i+1}^{1} \cup \cdots \cup \mathcal{N}_{i+1}^{2 l},
\end{aligned}
$$

The first equality holds, since $\mathcal{N}_{i}=\bigcup_{j=1}^{l} \mathcal{N}_{i}^{j}$ and $\hat{\mathcal{Z}}=$ $\hat{\mathcal{Z}}^{1} \cup \hat{\mathcal{Z}}^{2}$ by assumption and due to Lem. 5, respectively. The second and fourth equality hold, because $\tilde{S}(\mathcal{A} \cup \mathcal{B})=$ $\left[\begin{array}{ll}\hat{A} & \hat{b}\end{array}\right]^{-1}(\mathcal{A} \cup \mathcal{B})=\left[\begin{array}{ll}\hat{A} & \hat{b}\end{array}\right]^{-1} \mathcal{A} \cup\left[\begin{array}{ll}\hat{A} & \hat{b}\end{array}\right]^{-1} \mathcal{B}$ for arbitrary sets $\mathcal{A}, \mathcal{B} \subset \mathbb{R}^{n}$. The third equality results with the distributive law. The last equality results by substituting (56), (57).

The following proposition summarizes how exact nullcontrollable sets can be calculated for bilinear systems based on Lemmas 3, 5 and 6.

Proposition 2: Assume the bilinear system (1), (2) satisfies Assumption 1. Let $i^{*} \in \mathbb{N}, i^{*}>0$ be arbitrary. Then, the null-controllable set $\mathcal{N}_{i^{*}}$ can be computed with Alg. 1 .

Algorithm 1: Null-controllable set computation.

1 choose $c \in \mathbb{R}^{n}$ such that (15), (16) hold

2 calculate transformation matrix $M$ according to (18)

3 define linearized system by $\hat{A}=M^{-1} \tilde{A} M$ and $\hat{b}=M^{-1} \tilde{b}$

4 compute $H_{\hat{z}}^{(+)}, d_{\hat{z}}^{(+)}, H_{\hat{z}}^{(-)}$and $d_{\hat{z}}^{(-)}$according to (42), (43)

5 define $\hat{\mathcal{Z}}^{1}$ and $\hat{\mathcal{Z}}^{2}$ according to (40), (41) and set $\mathcal{N}_{0}^{1}=\{0\}$

6 for $i=0$ to $i^{*}-1$ do

7 for $j=1$ to $l=2^{i}$ do

$8 \quad$ calculate $\mathcal{N}_{i+1}^{2 j-1}$ and $\mathcal{N}_{i+1}^{2 j}$ according to (56), (57)

$9 \quad$ set $\mathcal{N}_{i+1}=\bigcup_{j=1}^{l} \mathcal{N}_{i+1}^{2 j-1} \cup \mathcal{N}_{i+1}^{2 j}$.

10 return sets $\mathcal{N}_{i^{*}}^{1}, \ldots, \mathcal{N}_{i^{*}}^{2 l}$ that describe $\mathcal{N}_{i^{*}}$ and terminate

Proof of Prop. 2: Assumption 1 guarantees the existence of an output (13) such that the exact linearization can be carried out. An appropriate $c \in \mathbb{R}^{n}$ is selected in line 1 of Alg. 1 . The linearized system (19), which applies for all $x \in \mathbb{R}^{n}$ and $u \in \mathcal{U}$ according to Lem. 3 , is defined in lines 2 and 3 . Lines 4 and 5 implement the constraints $\hat{\mathcal{Z}}=\hat{\mathcal{Z}}^{1} \cup \hat{\mathcal{Z}}^{2}$ from Lem. 5. The null-controllable sets $\mathcal{N}_{1}, \ldots, \mathcal{N}_{i^{*}}$ are computed with the procedure introduced in Lem. 6 in lines 6-10.

Remark 2: Alg. 1 results in $l:=2^{i^{*}}$ convex regions. This exponential growth of the number of regions cannot be avoided, but mitigated by fusing regions to form larger convex regions. Formally, this results in an optimization problem of the form

$$
\begin{aligned}
& \min _{\tilde{l}, \tilde{\mathcal{N}}_{i}^{1}, \ldots, \tilde{\mathcal{N}}_{i}^{\tilde{l}}} \tilde{l} \quad \text { s.t. } \quad \bigcup_{j=1}^{\tilde{l}} \tilde{\mathcal{N}}_{i}^{j}=\bigcup_{j=1}^{l} \mathcal{N}_{i}^{j}, \\
& \tilde{\mathcal{N}}_{i}^{j} \text { convex for } j=1, \ldots, \tilde{l} \text {. }
\end{aligned}
$$

It is generally not possible to solve (59) exactly. However, there exists algorithms for an approximate solution [9].

Remark 2 results in a significant simplification in Sect. V.

\section{NUMERICAL EXAMPLES}

We present two examples. We begin with a onedimensional system to illustrate the convex subsets $\hat{\mathcal{Z}}^{1}$ and $\hat{\mathcal{Z}}^{2}$ and the constraints $\hat{\mathcal{Z}}=\hat{\mathcal{Z}}^{1} \cup \hat{\mathcal{Z}}^{2}$ of the transformed linearized system (19). Moreover, the computation of the null-controllable sets $\mathcal{N}_{i}$ can be analyzed in detail for such a simple example. Subsequently, we study a two-dimensional example and illustrate the non-convexity of the sets $\mathcal{N}_{i}$. For both examples, we consider the constraints

$$
\mathcal{X}=\left\{x \in \mathbb{R}^{n} \mid\|x\|_{\infty} \leq 2\right\} \text { and } \mathcal{U}=\left\{u \in \mathbb{R} \mid\|u\|_{\infty} \leq 1\right\}
$$

and evaluate the null-controllable set $\mathcal{N}_{i^{*}}=\mathcal{N}_{12}$, where $i^{*}=$ 12 was chosen more or less arbitrarily.

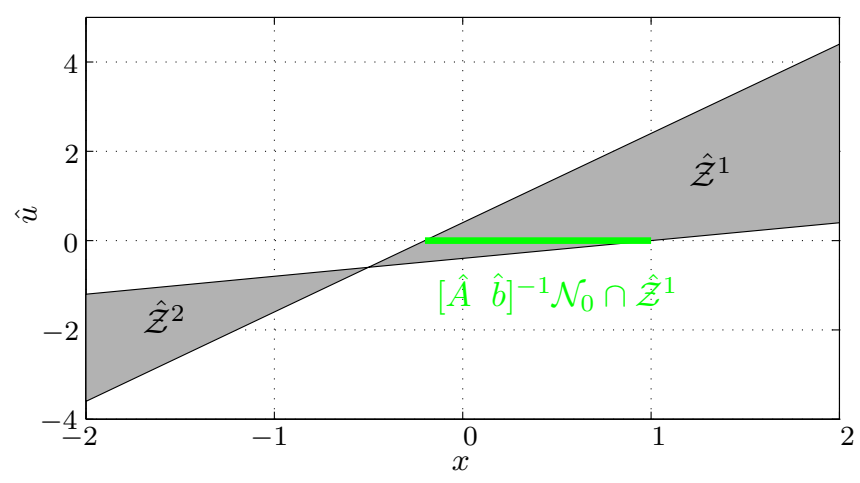

Fig. 1. Sets $\hat{\mathcal{Z}}^{1}$ and $\hat{\mathcal{Z}}^{2}$ for example 1 . The set $\left[\begin{array}{ll}\hat{A} & \hat{b}\end{array}\right]^{-1} \mathcal{N}_{0} \cap \hat{\mathcal{Z}}^{1}$, which is required in step $k=0$ of the recursion (56) $-(57)$, is marked in green.

Example 1: Consider the bilinear system (1), (2) with the parameters $A=1.2, b=0.4$ and $N=0.8$. Note that condition (21) holds, which is necessary for the existence of an appropriate linear output. The choice $c=1.0$ defines an output (13) such that the resulting SISO system has relative degree $r=n=1$. The transformation matrix $M$ defined in (18) reads $M=c^{T}=1.0$. The system matrices of the linearized system (19) read $\hat{A}=0.0$ and $\hat{b}=1.0$.

The sets $\hat{\mathcal{Z}}^{1}$ and $\hat{\mathcal{Z}}^{2}$ defined in (40)-(43), where

$$
H_{\tilde{z}}^{( \pm)}=\left[\begin{array}{rr}
1.0 & 0.0 \\
-1.0 & 0.0 \\
\mp 0.8 & 0.0 \\
\mp 1.2 \mp 0.8 & \pm 1.0 \\
\pm 1.2 \mp 0.8 & \mp 1.0
\end{array}\right], \quad d_{\tilde{z}}^{( \pm)}=\left[\begin{array}{r}
2.0 \\
2.0 \\
\pm 0.4 \\
\pm 0.4 \\
\pm 0.4
\end{array}\right]
$$

in this example, are visualized in Fig. 1. Algorithm 1 yields the null-controllable sets listed on the 1.h.s. in Tab. I. Since the example is one-dimensional, the sets $\mathcal{N}_{i}$ are intervals, which we denote by $\mathcal{N}_{i}=\left[\underline{\xi}_{i}, \bar{\xi}_{i}\right]$. Consider the first step

$$
\left[\begin{array}{ll}
\hat{A} & \hat{b}
\end{array}\right]^{-1} \mathcal{N}_{0}=\left[\begin{array}{ll}
0.0 & 1.0
\end{array}\right]^{-1}\{0\}=\left\{\hat{z} \in \mathbb{R}^{2} \mid \hat{z}_{2}=\hat{u}=0\right\}
$$

for illustration. The intersection $\left[\begin{array}{ll}\hat{A} & \hat{b}\end{array}\right]^{-1} \mathcal{N}_{0} \cap \hat{\mathcal{Z}}^{1}$ is highlighted in Fig. 1 in green. The projection of this line segment according to (56) results in $\mathcal{N}_{1}=[-0.2,1.0]$, which is the result listed in Tab. I. An analysis of the subsequent 
steps reveals that the upper bound $\bar{\xi}_{i}$ quickly converges to the bound $\bar{x}=2$ of the state constraints $\mathcal{X}=[\underline{x}, \bar{x}]$. In contrast, the lower bound $\underline{\xi}_{i}$ tends towards $-0.4>\underline{x}$. This is reasonable, since initial states $x_{0} \leq-0.4$ cannot be steered to the origin. For example, consider $x(0)=-0.4$, which results in $x(1)=-0.48+0.08 u(0)$. Obviously, even choosing the largest admissible control action $u(0)=1$ does not result in convergence to the origin.

TABLE I

NUMERICAL RESULTS FOR EXAMPLES 1 (LEFT) AND 2 (RIGHT).

\begin{tabular}{rc}
$i$ & $\mathcal{N}_{i}$ \\
\hline \hline 0 & {$[0.0000,0.0000]$} \\
1 & {$[-0.2000,1.0000]$} \\
2 & {$[-0.3000,2.0000]$} \\
5 & {$[-0.3875,2.0000]$} \\
12 & {$[-0.3999,2.0000]$}
\end{tabular}

\begin{tabular}{rrr}
$i$ & $l=2^{i}$ & $\tilde{l}$ \\
\hline \hline 0 & 1 & 1 \\
1 & 2 & 2 \\
2 & 4 & 4 \\
5 & 32 & 13 \\
12 & 4096 & 18
\end{tabular}

Example 2: Consider the bilinear system (1)-(2) with the parameters

$$
A=\left[\begin{array}{ll}
1.12 & 0.54 \\
0.76 & 0.92
\end{array}\right], b=\left[\begin{array}{r}
0.5 \\
-1.0
\end{array}\right], N=\left[\begin{array}{rr}
0.4 & -0.6 \\
-0.8 & 1.2
\end{array}\right] .
$$

It is easy to prove that condition (21) is satisfied and that $c^{T}=\left[\begin{array}{ll}2.0 & 1.0\end{array}\right]$ defines an appropriate output (13). This choice results in

$$
M=\left[\begin{array}{ll}
2.0 & 1.0 \\
3.0 & 2.0
\end{array}\right], \hat{A}=\left[\begin{array}{rr}
6.0 & 4.0 \\
-9.0 & -6.0
\end{array}\right], \hat{b}=\left[\begin{array}{r}
-1.0 \\
2.0
\end{array}\right] .
$$

The sets $\hat{\mathcal{Z}}^{1}$ and $\hat{\mathcal{Z}}^{2}$ can be defined according to (40)(43). Finally, evaluating the null-controllable sets $\mathcal{N}_{1}$ through $\mathcal{N}_{12}$ according to Alg. 1 yields the null-controllable region shown in Fig. 2. In contrast to the one-dimensional example above, the sets $\mathcal{N}_{i}$ are non-convex for every $i>0$. The sets $\mathcal{N}_{i}$ are therefore represented as a union of convex sets $\mathcal{N}_{i}^{j}$ as described in Sect. IV. The example can also serve as an illustration of the reduction of the number of the $\mathcal{N}_{i}^{j}$ mentioned in Rem. 1. In step $i=12$, for example, the number of regions can be reduced from $l=2^{i}=4096$ to $\tilde{l}=18$ (see r.h.s. of Tab. I for additional values).

In order to verify the computed null-controllable region, we calculated an approximation of the infeasible set, i.e. the complement of the maximal null-controllable set $\mathcal{N}_{\infty}:=$ $\lim _{i \rightarrow \infty} \mathcal{N}_{i}$. We used an algorithm introduced in [11] for this purpose, which is based on a sequential hyperrectangular partition of the state space. The outer approximation of the infeasible set, which is shown in red in Fig. 2, confirms the result found for the null-controllable set.

\section{CONCLUSIONS}

We presented a method for the exact computation of null-controllable sets for bilinear systems with input and state constraints. The proposed approach is based on an exact linearization of the dynamical system, a corresponding transformation for the constraints, and the application of recursive methods for the calculation of null-controllable sets for linear constrained systems. The transformation of the constraints results in non-convex constraints even if the

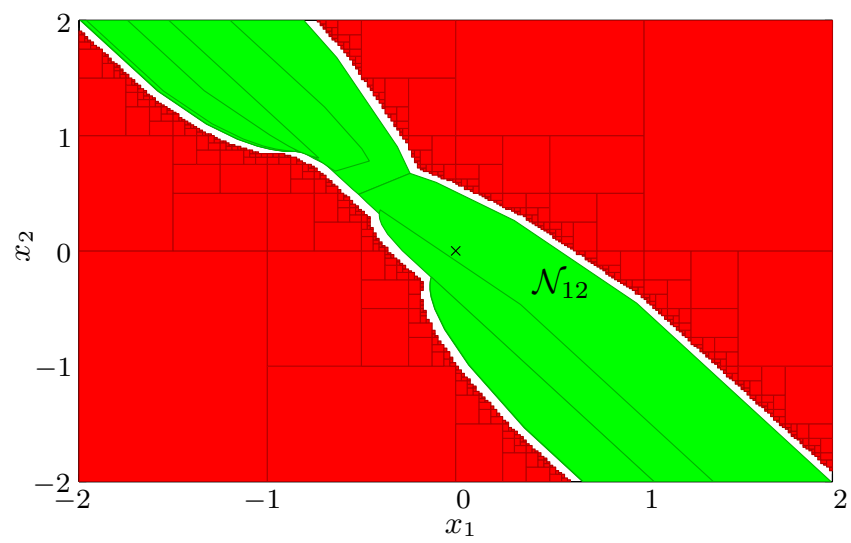

Fig. 2. Exact null-controllable set $\mathcal{N}_{12}$ for example 2 shown in green. Inner approximation of the infeasible set based on [11] shown in red.

bilinear system was originally subject to convex input and state constraints. However, we showed that the transformed constraints can be represented by two convex sets. This is essential for an efficient computer-based evaluation of the null-controllable sets.

We illustrated the approach with two examples. For the more complicated two-dimensional example, we found, as anticipated, a non-convex shape of the null-controllable set. We verified our results with outer approximations of the nullcontrollable set.

We pointed out that the approach in the current form only applies to a small class of bilinear systems (1), specifically single-input systems with $\operatorname{rk}(B)=1$, where $B=\left[\begin{array}{ll}b & N\end{array}\right]$. Future work has to address the extension to multi-input systems and output constraints.

\section{REFERENCES}

[1] J. M. Bravo, D. Limon, T. Alamo, and E. F. Camacho. On the computation of invariant sets for constrained nonlinear systems: An interval arithmetic approach. Automatica, 41(9):1583-1589, 2005.

[2] C. Bruni, G. DiPillo, and G. Koch. Bilinear systems: An appealing class of "nearly linear" systems in theory and applications. IEEE Transactions on Automatic Control, 19(4):334-348, 1974.

[3] C.-T. Chen. Linear System Theory and Design. Oxford University Press, New York, 1998.

[4] D. Elliot. Bilinear Control Systems. Springer, 2009.

[5] P.O. Gutman and M. Cwikel. An algorithm to find maximal state constraint sets for discrete-time linear dynamical systems with bounded control and states. IEEE Transactions on Automatic Control, 32(3):251-253, 1987.

[6] M.L.J. Hautus. Stabilization, controllability and observability of linear autonomous systems. Proc. of the Koninklijke Nederlandse Akademie van Wetenschappen, 73(5):448-455, 1970.

[7] A. Isidori. Nonlinear Control Systems. Springer, 1995.

[8] S.S. Keerthi and E.G. Gilbert. Computation of minimum-time feedback control laws for discrete-time systems with state-control constraints. IEEE Transactions on Automatic Control, 32(5):432-435, 1987.

[9] M. Kvasnica, P. Grieder, M. Baotić and M. Morari. Multi-Parametric Toolbox (MPT). In Proc. of 7th International Workshop on Hybrid Systems - Computation and Control, 448-462, 2004. (The function merge was used for (59).)

[10] R.T Rockafellar. Convex Analysis. Princeton University Press, 1972.

[11] M. Schulze Darup and M. Mönnigmann. Low complexity suboptimal explicit NMPC. In Proc. of 4th IFAC Nonlinear MPC Conference, 406-411, 2012. (Alg. parameters set to $\underline{d}_{x}=\bar{d}_{x}=16$ and $d_{u}=8$.) 